\title{
Metastatic solitary fibrous tumor of the pancreas in a patient with Doege-Potter syndrome
}

\author{
Xiaohui Qian ${ }^{1,2,3,4,5}$, Dongkai Zhou ${ }^{1,2,3,4,5}$, Bingqiang Gao ${ }^{1,2,3,4,5}$, Weilin Wang ${ }^{1,2,3,4,5}$ \\ ${ }^{1}$ Department of Hepatobiliary and Pancreatic Surgery, The Second Affiliated Hospital, Zhejiang University School of Medicine, Hangzhou 310009 , \\ China; ${ }^{2}$ Key Laboratory of Precision Diagnosis and Treatment for Hepatobiliary and Pancreatic Tumor of Zhejiang Province, Hangzhou 310009 , \\ China; ${ }^{3}$ Research Center of Diagnosis and Treatment Technology for Hepatocellular Carcinoma of Zhejiang Province, Hangzhou 310009, China; \\ ${ }^{4}$ Clinical Medicine Innovation Center of Precision Diagnosis and Treatment for Hepatobiliary and Pancreatic Disease of Zhejiang University, \\ Hangzhou 310009, China; ${ }^{5}$ Clinical Research Center of Hepatobiliary and Pancreatic Diseases of Zhejiang Province, Hangzhou 310009 , China \\ Correspondence to: Weilin Wang. Department of Hepatobiliary and Pancreatic Surgery, The Second Affiliated Hospital, Zhejiang University School of \\ Medicine, No. 88 Jiefang Road, Hangzhou 310009, China. Email: wam@zju.edu.cn.
}

Submitted Nov 07, 2019. Accepted for publication Nov 14, 2019.

doi: 10.21037/hbsn.2019.12.01

View this article at: http://dx.doi.org/10.21037/hbsn.2019.12.01

A 46-year-old man was admitted to our hospital with a 6-month history of recurrent hypoglycemia with an unknown cause. Four months back, he was diagnosed with a complex ulcer in the stomach and duodenum in a local hospital. Physical examination revealed that the liver was swollen with a hard texture and located $5 \mathrm{~cm}$ below the costal margin of the abdomen. On admission, his random blood glucose level was $1.6 \mathrm{mmol} / \mathrm{L}$, and routine laboratory tests, including those for liver enzymes, showed normal findings. The levels of tumor markers, including carcinoembryonic antigen, alpha-fetoprotein, carbohydrate antigen 19-9, and neuron-specific enolase, were normal. Magnetic resonance imaging (MRI) of the brain and pituitary gland showed no abnormalities. However, abdominal MRI revealed solid components in the pancreas and multiple masses on the liver (Figure 1A,B,C). We first considered functional neuroendocrine cancer of the pancreas with multiple liver metastases. For blood glucose control, the patient first received multiple transarterial chemoembolization (TACE) sessions to reduce the tumor burden. This resulted in amelioration of the symptoms of hypoglycemia, although they occasionally manifested. Whole-body fluorodeoxyglucose (FDG) positron emission tomography (PET)/computed tomography (CT) revealed a slightly hypodense lesion measuring $7.0 \times 6.1 \mathrm{~cm}^{2}$ in diameter in the body of the pancreas. The lesion exhibited significantly different degrees of FDG uptake [Figure 1D; maximum standardized uptake (SUVmax): 4.74].
In addition, multiple, slightly hypodense lesions exhibiting incomplete lipiodol uptake and remarkable levels of FDG uptake (Figure 1E, SUVmax: 4.79-5.04) were observed in the liver. FDG uptake in the pleura, extremities, and retroperitoneum was unremarkable. Distal pancreatectomy and left lateral hepatectomy were performed to reduce the size of the tumors, maintain a stable blood glucose level, and establish a definitive diagnosis. Postoperative histopathology showed a spindle cell tumor. The tumor cells were mostly heterotypic, with a mitotic count of approximately $4-5 / 10$ high power fields (Figure $1 F$ ). Local infarction was observed. Immunohistochemistry showed diffuse STAT6 positivity; CD34 and Bcl-2 positivity; a Ki67 index of 10\%; and cytokeratin, S-100, and smooth muscle actin negativity (Figure 1G,H,I). A diagnosis of solitary fibrous tumor (SFT) of the pancreas was made based on the histological and immunohistochemical findings. The patient's postoperative recovery was uneventful, and he was discharged. Blood sugar fluctuations within the normal range were randomly detected. Although multiple recurrences and metastases developed at 6 months after the surgery, the patient was alive at the 10-month follow-up.

SFT is a rare fibroblastic mesenchymal tumor that was first reported by Lemercier and Desseauve in 1931 (1). It mostly originates in the pleura. Extrapleural SFTs develop in deep soft tissue sites such as the extremities (2), and retroperitoneum (3). SFT of the pancreas is extremely rare, and no one accompany with paraneoplastic syndrome. 

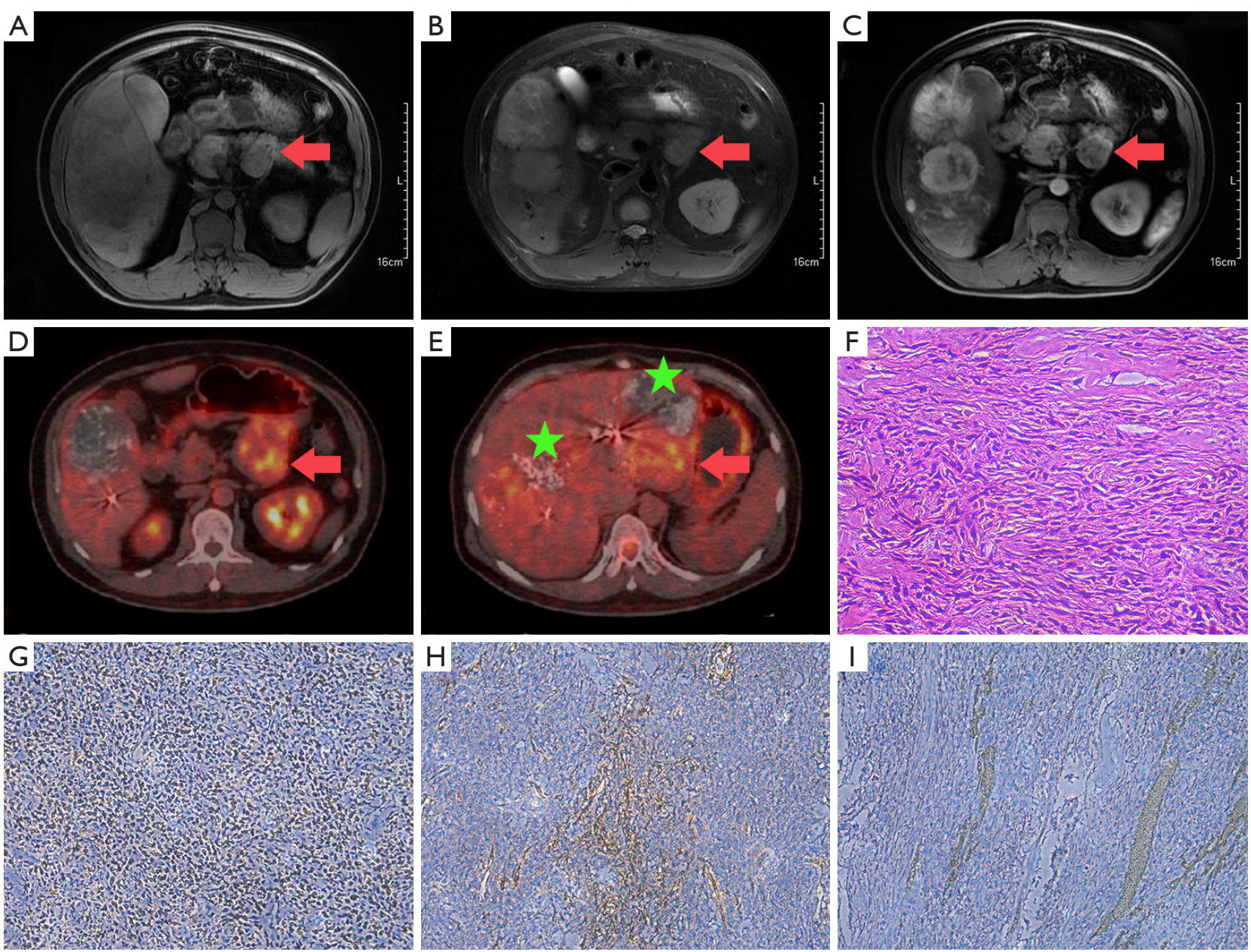

Figure 1 Imaging, histopathological, and immunohistochemical findings. (A,B,C) Magnetic resonance imaging: axial T1-weighted imaging shows a well-circumscribed mass in the pancreatic head (red arrow), with decreased signal intensity relative to that of the normal pancreas (A); axial T2-weighted imaging shows the mass (red arrow) with heterogeneous, slightly high signal intensity relative to that of the normal pancreas (B); axial contrast-enhanced imaging shows the mass (red arrow) with heterogeneous increased arterial phase enhancement (C); positron emission tomography/computed tomography $(\mathrm{D}, \mathrm{E})$ : the pancreatic mass can be observed with significantly different degrees of FDG uptake (D; red arrow; SUVmax: 4.74). Multiple, slightly hypodense lesions exhibiting incomplete lipiodol uptake (E; green stars) and remarkable levels of FDG uptake (E; red arrow; SUVmax: 4.79-5.04) are present in the liver; (F,G,H,I) histopathological and immunohistochemical findings: the mass is a spindle cell tumor rich in heterotypic cells (400×) (F); immunohistochemistry shows positive staining for STAT6 (200×) (G), CD34 (200×) (H), and Bcl-2 (200x) (I). SUVmax, maximum standardized uptake. FDG, fluorodeoxyglucose.

Doege and Potter first described a large intrathoracic mass with symptoms of hypoglycemia; since then, noninsulinoma hypoglycemia is referred to as Doege-Potter syndrome (DPS) (4). DPS often occurs as a paraneoplastic syndrome associated with various tumors such as hepatocellular carcinoma and gastrointestinal stromal tumors, and it is associated with approximately $4 \%$ of benign and malignant forms of SFT. To our knowledge, this is the first report of pancreatic SFT with multiple liver metastases in a patient with DPS.

We searched the PubMed database for all reported cases of pancreatic SFTs and found that most were identified in the pancreatic head and body. SFTs normally exhibit specific imaging features because of the presence of alternating cellular (vascular) and paucicellular fibrous components. On contrast-enhanced images, the cellular components appear hypervascular, demonstrating increased enhancement and a well-developed vascular 
supply with large feeding vessels. The fibrous component accumulates the contrast agent over time and exhibits a progressive enhancement pattern (5). In addition, most large tumors show central degeneration and necrosis. Usually, the majority of SFTs exhibit a thin capsule with well-circumscribed margins, without invasion into the adjacent parenchyma. PET/CT findings for SFTs have not been well documented in the literature. A few case reports and small studies of pleural and extrapleural SFTs have shown no or mild FDG uptake in most tumors (6). Although most SFTs are benign, their behavior can be unpredictable, and assessment of the metastatic risk remains a debatable topic (7). In the present case, wholebody PET/CT showed SFTs with FDG uptake, and we could observe similar FDG uptake in the primary and metastatic lesions. Because increased cellularity and more aggressive biological behavior are now being observed in malignant SFTs, we believe that PET/CT has the potential to detect malignant SFTs, particularly in patients with DPS.

Surgical resection is the most effective curative treatment for primary tumors and prevents recurrent hypoglycemia (8). Complete tumor resection is the preferred treatment for avoiding SFT recurrence in cases of DPS. In some cases, paraneoplastic symptoms such as DPS can disappear quickly and completely after radical resection. In addition, paraneoplastic symptoms can be a sign of tumor recurrence, and they usually reappear within 24 months after resection (9). There are no guidelines for the treatment of metastatic or symptomatic nonresectable SFTs because of their rarity. SFTs are relatively chemoresistant, and diverse chemotherapy regimens are described with differing efficacies. Recently, some studies showed that targeted therapy (e.g., Akt/mTOR pathway) may be useful (10). The role of radiotherapy also remains unclear, although it is considered beneficial as an adjuvant treatment with surgery. Our patient received multiple TACE sessions and surgery in succession to reduce the tumor burden. Although the lesions recurred 6 month later, the regimen appears to be useful for the palliative treatment of metastatic or nonresectable SFTs.

In summary, we reported the first case of a metastatic pancreatic SFT associated with DPS. SFT should be considered as a differential diagnosis for hypervascular pancreatic lesions, particularly those manifesting with refractory hypoglycemia. PET/CT may be useful for assessing the biological behavior and metastatic risk of SFTs, while TACE and partial surgery may be useful palliative therapies to prolong survival and improve the quality of life.

\section{Acknowledgments}

None.

\section{Footnote}

Conflicts of Interest: The authors have no conflicts of interest to declare.

Ethical Statement: The authors are accountable for all aspects of the work in ensuring that questions related to the accuracy or integrity of any part of the work are appropriately investigated and resolved. Written informed consent was obtained from the patient for publication of this manuscript and any accompanying images.

\section{References}

1. Lemercier JP, Desseauve J. Primary Neoplasms of the Pleura. Gaz Med Fr 1963;70:3047-54.

2. Anders JO, Aurich M, Lang T, et al. Solitary fibrous tumor in the thigh: review of the literature. J Cancer Res Clin Oncol 2006;132:69-75.

3. Kume M, Komori K, Inoguchi H, et al. Solitary fibrous tumor in the retroperitoneal space: report of a case. Surg Today 2002;32:1099-101.

4. Oana S, Matsuda N, Sibata S, et al. A case of a "wandering" mobile solitary fibrous tumor occurring in the pancreas. Clin J Gastroenterol 2017;10:535-40.

5. Manning MA, Paal EE, Srivastava A, et al. Nonepithelial Neoplasms of the Pancreas, Part 2: Malignant Tumors and Tumors of Uncertain Malignant Potential From the Radiologic Pathology Archives. Radiographics 2018;38:1047-72.

6. Parghane RV, Agrawal K, Mittal BR, et al. 68Ga DOTATATE PET/CT imaging of a solitary fibrous tumor of the nasopharynx. Clin Nucl Med 2014;39:e382-3.

7. D'Amico FE, Ruffolo C, Romano M, et al. Rare Neoplasm Mimicking Neuoroendocrine Pancreatic Tumor: A Case Report of Solitary Fibrous Tumor with Review of the Literature. Anticancer Res 2017;37:3093-7.

8. Sheng Q, Xu W, Liu J, et al. Pancreatic solitary fibrous tumor in a toddler managed by pancreaticoduodenectomy: a case report and review of the literature. Onco Targets 
Ther 2017;10:1853-8.

9. Kalebi AY, Hale MJ, Wong ML, et al. Surgically cured hypoglycemia secondary to pleural solitary fibrous tumour: case report and update review on the Doege-Potter

Cite this article as: Qian X, Zhou D, Gao B, Wang W. Metastatic solitary fibrous tumor of the pancreas in a patient with Doege-Potter syndrome. Hepatobiliary Surg Nutr 2020;9(1):112-115. doi: 10.21037/hbsn.2019.12.01 syndrome. J Cardiothorac Surg 2009;4:45.

10. de Boer J, Jager PL, Wiggers T, et al. The therapeutic challenge of a nonresectable solitary fibrous tumor in a hypoglycemic patient. Int J Clin Oncol 2006;11:478-81. 